\title{
Analyse Exploratrice Des Modèles De Référence De L'évaluation De La Performance De La Chaine Logistique
}

\author{
El Gharbaoui Mohammed, Doctorant \\ Laboratoire de recherche en sciences de Gestion, ENCG Kénitra, \\ Université Ibn Tofail, Maroc
}

Doi:10.19044/esj.2020.v16n13p303 URL:http://dx.doi.org/10.19044/esj.2020.v16n13p303

\section{Resume}

L'intérêt pour l'évaluation de la performance de la chaine logistique a considérablement augmenté au cours des trois dernières décennies avec de multiples recherches menées. Cet article veut examiner les modèles de référence $\left(\mathrm{SCOR}^{\circledR}, \mathrm{BSC}, \mathrm{ECOGRAI}\right.$ et $\left.\mathrm{ABC}\right)$ en mettant en évidence leurs apports et leurs limites dans l'évaluation de la performance de la chaine logistique. Ces modèles sont classés en trois approches principales : Approche axée sur les processus, approche axée sur les perspectives et approche axée sur les niveaux hiérarchiques. En effet, ces modèles reposent sur la conception d'un système d'indicateurs de performance multicritères et multidimensionnels pour assurer la mesure de la performance et associent ses indicateurs à des activités et à des centres de décisions tout au long de la chaine logistique permettant une évaluation de la performance. Cet article révèle que les modèles exploités sont fragilisés par leur incapacité à identifier les sources de performance et de non-performance dans la chaine logistique et par conséquent leur insuffisance dans l'élaboration d'un plan d'action résultant de l'évaluation de la performance de la chaine logistique. Ainsi, les contributions futures à la recherche sur l'évaluation des performances de la chaine de performance sont possibles et souhaitables pour surpasser les limites des modèles de référence actuels.

Mots-clés: La chaine logistique, la performance, modèle d'évaluation 


\title{
An Exploratory Analysis of Reference Models for Supply Chain Performance Evaluation
}

\author{
El Gharbaoui Mohammed, Doctorant \\ Laboratoire de recherche en sciences de Gestion, ENCG Kénitra, \\ Université Ibn Tofail, Maroc
}

\begin{abstract}
Interest in the topic of supply chain performance assessment has increased considerably over the last three decades and considerable research has been conducted in this area. The objective of this paper is to review the benchmark models $\left(\mathrm{SCOR}^{\circledR}, \mathrm{BSC}, \mathrm{ECOGRAI}\right.$ and $\left.\mathrm{ABC}\right)$ to highlight their contributions and limitations in the contribution of supply chain performance assessment. These models can be classified into three main approaches: process-based approach, perspective-based approach and hierarchical level approach. These models are based on the design of a system of multi-criteria and multidimensional performance indicators for performance measurement and link these indicators to activities and decision centres along the supply chain to enable performance assessment. This article reveals that the models used are weakened by their inability to identify the sources of performance and non-performance in the supply chain and consequently their inadequacies in the development of an action plan to result from the evaluation of supply chain performance. Thus, future contributions to research on supply chain performance assessment are possible and desirable to overcome the limitations of current benchmarking models.
\end{abstract}

Keywords: Supply chain, performance, evaluation model

\section{Introduction}

La performance et son évaluation peuvent être considérées comme des conditions nécessaires au succès d'une organisation, elles constituent un champ de recherche conséquent en sciences de gestion, orienté par une forte demande des dirigeants et gestionnaires des entreprises pour se doter de meilleures pratiques pour améliorer la performance globale de l'entreprise (Salgado, 2013).

Aujourd'hui, la gestion de la chaine logistique, nommée chez les anglophones supply chain management, joue un rôle stratégique dans le processus de la recherche de la performance entamé par toute entreprise. La 
chaine logistique, constituée de l'ensemble des acteurs existants sur la chaine de valeur de l'entreprise pour la création de la richesse, a besoin d'un ensemble des activités pour conduire et améliorer l'ensemble du cheminement des flux physiques, financiers et informationnels, du premier des fournisseurs jusqu'aux clients finaux/consommateurs (Cheyroux, 2003).

La gestion de la chaine logistique s'appuie sur l'optimisation du triangle (coûts, qualité, délais) pour assurer une meilleure performance de l'entreprise, l'évaluation de la performance de la chaine logistique permet de fournir les informations nécessaires et utiles pour les dirigeants au niveau stratégique, tactique et opérationnel pour apprécier le niveau de performance de l'entreprise et identifier les actions possibles pour son amélioration (Kusrini et al., 2014), d'autre part, le pilotage et l'amélioration de la performance de la chaine logistique ont été jugés comme une fonction complexe par les dirigeants (Morgan, 2007).

(Gunasekaran et al., 2004) ont insisté que l'évaluation de la performance de la chaine logistique est un élément essentiel dans sa gestion, (Lai et al., 2002) affirme que l'absence d'un modèle adapté pour l'évaluation de la performance de la chaine logistique peut être une condition majeure de l'échec de la gestion de la chaine logistique. Pour (Abu-Suleiman et al., 2004), l'importance de l'adoption d'un modèle d'évaluation de la performance de la chaine logistique est justifiée par le fait que les actions pour améliorer la performance ne peuvent pas être déterminées sans l'appui d'un modèle/système d'évaluation de la performance et que l'évaluation de la performance fournit, à la fois, une aide à la décision et un retour d'expérience accessible aux dirigeants pour le pilotage des actions d'amélioration de la performance et pour la communication à l'ensemble des acteurs de la chaine logistique.

La prise en compte du concept de la performance de la chaine logistique dans le domaine de la recherche a été sollicitée par un grand nombre de publications, ainsi, de l'année 1986 jusqu'au premier trimestre de $2019^{13}$, 1788 publications scientifiques ont contribué à l'enrichissement autour de ce thème. L'expression "performance de la chaine logistique » a été citée dans les publications scientifiques près de 30000 fois.

En l'absence d'un modèle unifié d'évaluation de la performance de la chaine logistique faisant l'unanimité de chercheurs; ce travail est une contribution à l'étude exploratrice et critique de différents modèles identifiés dans le champ de l'évaluation de la performance de la chaine logistique. Son but ultime est de fournir une base d'information pour les utilisateurs désireux

\footnotetext{
${ }^{13}$ Donnée consultée le 05/03/19 sur la base de données bibliographique «Web of Science » en utilisant le terme « Supply Chain Performance».
} 
d'adopter ou de concevoir un modèle d'évaluation de la performance adapté à leur besoin.

L'objectif de ce travail est de présenter, à l'aide d'une revue de littérature, une analyse critique sur les modèles de références de l'évaluation de la chaine logistique choisis par une lecture bibliographie afin de déceler les différents aspects liés à leur utilisation en management des chaines logistiques. Le présent article est organisé comme suit : après la brève introduction, la première section expose d'abord le champ conceptuel de l'évaluation de la performance de la chaine logistique, ensuite la deuxième section interprète les différents modèles d'évaluation de la performance de la chaine logistique, et enfin la troisième section présente une comparaison entre les modèles de références exploités. La dernière section conclut en présentant les perspectives de ce travail.

\section{Le champ conceptuel de l'évaluation de la performance de la chaine logistique}

Historiquement, l'évaluation de la performance s'est longuement orientée vers un horizon financier où la création de la richesse des actionnaires est privilégiée (Lappide, 2002). Des indicateurs purement financiers (Chiffre d'affaires, Résultat, Retour sur investissement...) ont été largement utilisés pour servir de base à cette évaluation dans le sens financier.

Graduellement, les chercheurs ont penché vers la création d'indicateurs non financiers à associer aux indicateurs financiers pour l'évaluation de la performance. Le but ultime poursuivi étant la conception d'une évaluation multicritère et multidimensionnelle où les intérêts de toutes les parties prenantes (les dirigeants, les salariés, les actionnaires, les clients, les fournisseurs, l'environnement) sont intégrés (Finet, 2009).

Aussi, les recherches récentes dans le domaine de la performance s'intéressent à l'identification et à l'étude des déterminants de la performance dans le but de doter les dirigeants d'un maximum d'informations pour mettre en place une meilleure stratégie pour l'entreprise (Brulhart et Moncef, 2010).

Dans ce contexte, la gestion de la chaine logistique est devenue une source déterminante de la performance de l'entreprise dans la mesure où elle contribue à l'amélioration de sa compétitivité à travers la création de la valeur pour tous les acteurs de la chaine logistique (Waters \& Chartered Institute of Logistics and Transport in the UK, 2007)

L'enquête internationale «Service Supply Chain report 2018 ${ }^{14}$, menée par le groupe PricewaterhouseCoopers $(\mathrm{Pwc})^{15}$, indique une corrélation

\footnotetext{
${ }^{14}$ Consulté le 03/03/2019 sur le lien suivant : https://www.pwc.be/en/documents/20180326pwc-service-supply-chain-survey.pdf

15 Le groupe Pwc est un réseau d'entreprises spécialisées dans des missions d'audit, d'expertise comptable et de conseil à destination des entreprises sur 158 pays.
} 
directe entre la maturité de la gestion de la chaine logistique et les profits réalisés par l'entreprise, plus précisément, une maturité basique de la gestion de la chaine logistique peut améliorer la marge brute de l'entreprise de $15 \%$, dans le cas d'une maturité avancée, l'amélioration de la marge brute est estimée à $50 \%$.

Toujours, selon le rapport de l'enquête du groupe Pwc, une maturité basique de la gestion de la chaine logistique correspond à une gestion réactive à la demande des clients où le prix du produit est basé sur les coûts et les fonctions de l'entreprise (Production, transport....) travaillent de façon quasi indépendante chacune avec ses objectifs. Quant à une maturité avancée, qui correspond à une gestion de toute la chaine de la valeur inclut les fournisseurs et les clients où le prix du produit est basé sur la valeur et les fonctions de l'entreprise travaillent en parfaite collaboration avec des objectifs en communs.

Par ailleurs, le niveau de la maturité de la gestion de la chaine logistique est un critère déterministe du modèle de l'évaluation de la performance de la gestion de la chaine logistique (Estampe et al., 2013).

Pour la suite de cet article, des définitions de l'évaluation de la performance, la chaine logistique, l'évaluation de la performance de la gestion de la chaine logistique sont présentées afin de mieux cerner le périmètre de ce travail.

(Salgado, 2013) indique que la performance se définit par des indicateurs multicritères et multi acteurs et non pas par une mesure en quelque sorte unique, il rejoint (Lebas, 1995) pour affirmer que la performance est une notion à la fois floue, multiforme, multidimensionnelle, contingente, et difficile à définir et à mesurer. (Lorino, 2003) et (Derujinsky et al., 2011) précisent que la performance ne prenant réellement de sens que lorsqu'elle est instrumentale.

Ainsi, l'évaluation de la performance est une instrumentalisation du concept de la performance sur la base d'un processus structuré autour des activités qui peuvent être conçues sur la base du travail (Marr, 2006) et le cycle PDCA $^{16}$ [Qing-ling et al., 2008] comme suit :

\footnotetext{
${ }^{16}$ PDCA (Plan, Do, Check et Act, en anglais), les quatre piliers fondamentaux de la méthode : une démarche séquentielle divisée en quatre étapes ayant pour objectif d'améliorer continuellement la conduite d'un projet ou le fonctionnement de l'entreprise.
} 
Figure 1: Activités de l'évaluation de la performance

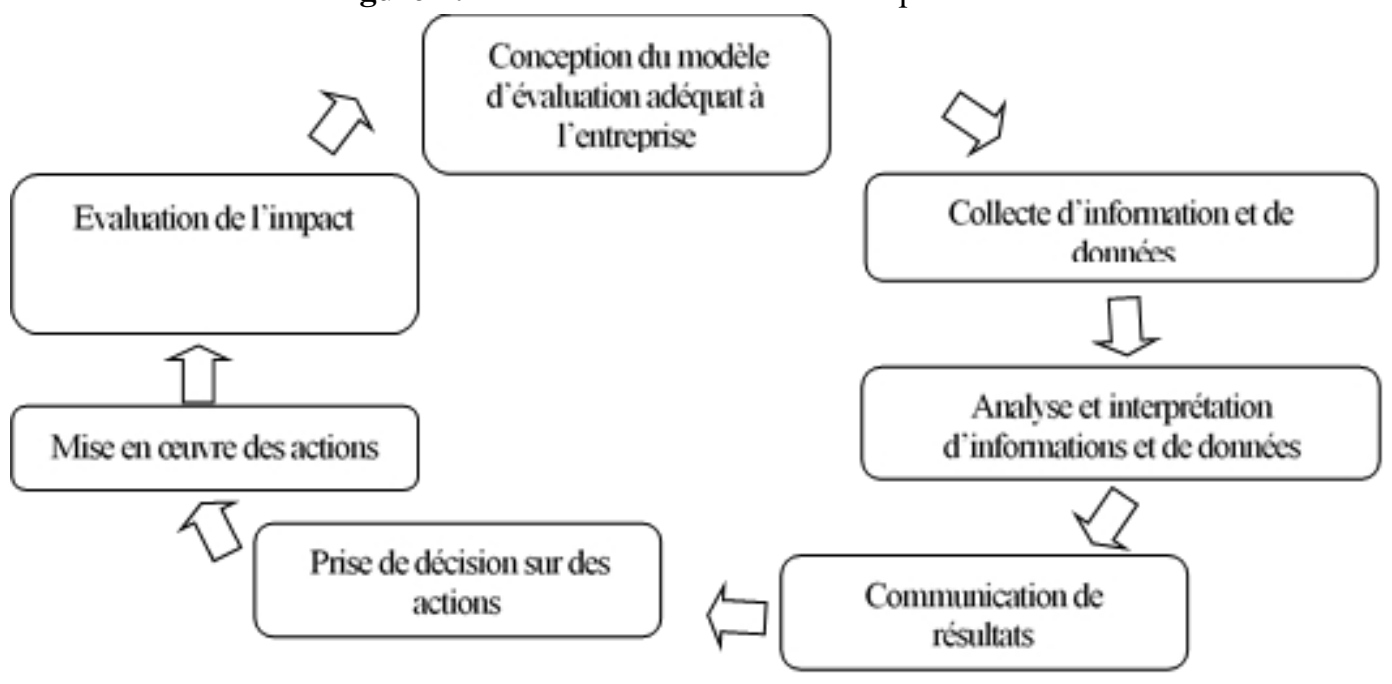

L'évaluation de la performance de la chaine logistique a été appréhendée dans le milieu académique dans la mesure où elle contribue à l'amélioration générale de la performance. Au début des années 90, les dirigeants ont commencé à avoir l'idée qu'il ne faut pas se contenter de chercher les sources/déterminants de la performance à l'intérieur de la chaine de valeur au sein de l'entreprise et qu'il est indispensable d'avoir une vue générale sur toute la chaine de la valeur qu'intègre les différents partenaires comme les fournisseurs et les clients finaux, aussi que leurs propres employés (Fitzgerald et al., 1991).

Pour (Ho et al., 2002), la chaine logistique se définit comme un réseau des organisations impliquées en collaboration, en amont et en aval, dans la création de la valeur, à travers des processus et des activités, pour remettre aux clients ultimes des produits et des services, ainsi la gestion de la chaine logistique se présente comme une gestion des processus et des activités associés aux flux matériels, informationnels et financiers qui assurent la réponse à un besoin de consommation (produit ou service) d'un client final à partir de la première demande adressée au fournisseur initial de l'entreprise.

Dans ce sens et selon (Holmberg, 2000), l'évaluation de la performance de la chaine logistique (cf. figure 2) est interprétée comme un système qui définit formellement le modèle de l'évaluation de la chaine logistique basé sur des objectifs, des mesures et des évaluations convenus d'un accord commun de tous les acteurs de la chaine logistique (de premier fournisseur au client final). 
Figure 2 : Illustration basique de la gestion de la chaine logistique et de son évaluation

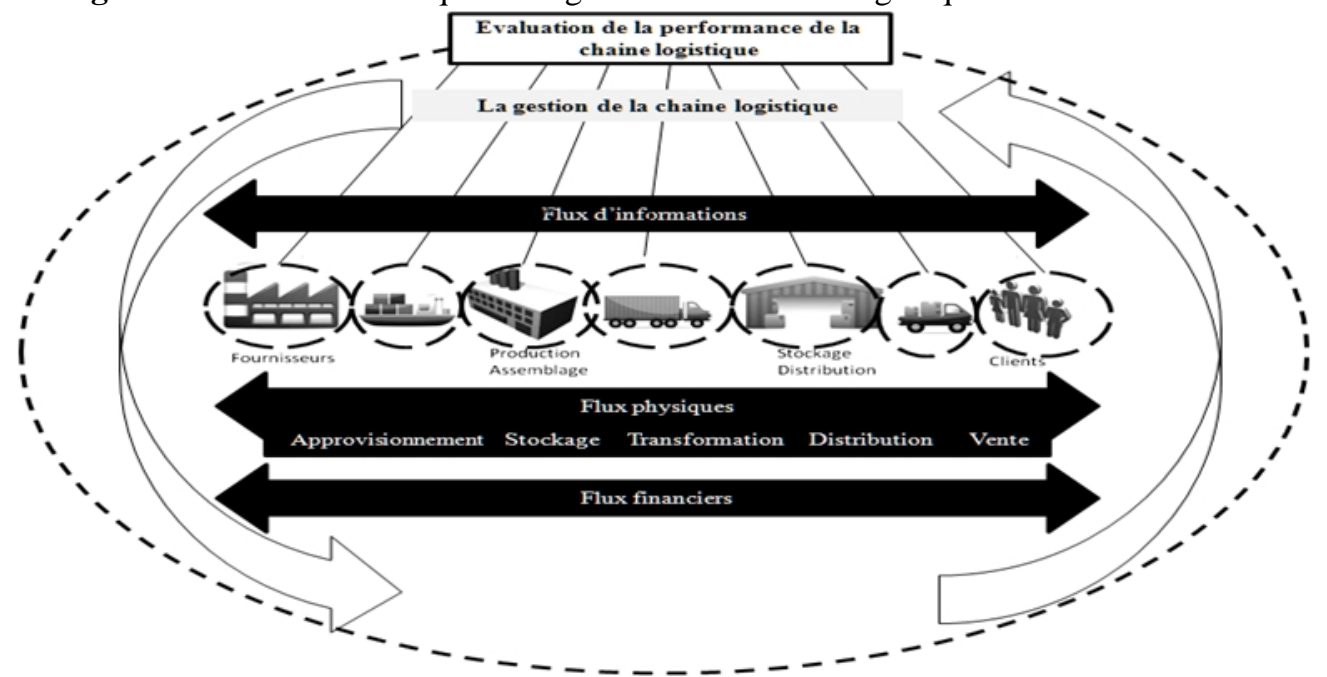

II. Les modèles d'évaluation de la performance de la chaine logistique

Dans le début des années 1990, (Lee et Billington, 1992) affirment qu'aucun modèle d'évaluation de la performance de la chaine logistique n'avait existé, l'évaluation de la performance se portait principalement sur un seul processus ou une seule organisation, mais graduellement, (Beamon, 1998) précise que l'accent a été mis sur l'évaluation de la performance de l'ensemble de la chaine logistique en tant qu'un système d'activités interdépendantes. Il existe aujourd'hui une variété de modèles tenant compte la particularité de la chaine logistique (Rafele, 2004).

En se basant sur le travail de (Neely et al., 1995) sur les critères d'éligibilité d'un modèle d'évaluation de la performance, (Kusrini et al., 2014) décrivent quatre critères spécifiques d'un modèle d'évaluation de la performance de la chaine logistique :

1. L'équilibre: le modèle doit tenir compte de l'ensemble de spécifications et mesures fournies par tous les acteurs de la chaine logistique. 2. L'intégration : le modèle doit couvrir l'ensemble de mesures de la chaine logistique.

3. La spécification : le modèle doit être spécifique par des mesures de performance axées sur les processus d'affaires créateurs de la valeur ajoutée. 4. La détermination: le modèle doit déterminer l'impact de contributions de l'ensemble d'acteurs de la chaine logistique sur la performance de la chaine logistique.

Les contributions (Gunasekaran et Kobu, 2007), (Ramaa et al., 2009) et (Avelar-Sosa et al., 2019) ont classifié les modèles sur la base des critères liés aux : 
- $\quad$ Perspectives de la performance

- Composantes de la performance (flexibilité, utilisation des ressources, innovation, qualité, la réactivité, la productivité et la fiabilité)

- $\quad$ Processus élémentaires de la chaine logistique

- Types de décisions dans l'entreprise (stratégique/opérationnelle/tactique)

- $\quad$ Natures de mesure : financier ou non-financier

- $\quad$ Types de mesure : quantitative ou qualitative

- Orientations de mesure: traditionnelle basée sur la fonction ou moderne basée sur la valeur

Les contributions, citées ci-dessus, ont souligné des modèles de références qui ont servi de base pour déclencher la conception d'autres modèles d'évaluation de la performance de la chaine logistique regroupés selon trois approches principales.

Cet article couvre ses trois approches en proposant le modèle de référence adéquat pour chacune des approches.

Tableau 1: les approches de l'évaluation de la performance de la chaine logistique

\begin{tabular}{|l|ll|}
\hline Approche & \multicolumn{2}{|l|}{ Modèle de référence cité } \\
\hline Approche axée sur les processus & - & ABC : Activety Based Costing \\
\hline \multirow{2}{*}{ Approche axée sur les perspectives } & - & BSC: Balanced ScoreCard; \\
& - & SCOR: Supply Chain Operation Reference \\
\hline Approche axée les niveaux hiérarchiques & - & ECOGRAI \\
\hline
\end{tabular}

Sur la base de ces critères, les modèles suivants ont été explorés et analysés :

\section{II.1. Le modèle $\mathrm{SCOR}^{\circledR}$}

Le modèle SCOR ${ }^{\circledR}$ (Supply Chain Operation Reference) est un modèle de référence développé en 1996 par l'organisation indépendante sans but lucratif : Supply Chain Council ${ }^{17}$, il permet de modéliser la chaine logistique pour avoir une vision globale sur les flux informatiques, physiques et financiers entre le fournisseur du fournisseur jusqu'au client du client d'une entreprise. Le modèle $\mathrm{SCOR}^{\circledR}$ propose une structure de référence de la chaine logistique subdivisée en cinq types de processus: planification, achat, fabrication, livraison et gestion des retours et pour chacun des processus, il introduit des indicateurs de performances (Huang et al., 2005).

Ainsi, le modèle $\mathrm{SCOR}^{\circledR}$ a deux vocations, la première permettant la modélisation des processus de la chaine logistique à l'aide de diagrammes et la seconde consiste à la mise en place d'indicateurs permettant l'évaluation de la performance de la chaine logistique.

${ }^{17}$ Supply chain operations reference model. Disponible sur : http://www.apics.org/sites/apicssupply-chain-council. 
Le modèle $\mathrm{SCOR}^{\circledR}$ identifie cinq attributs de la performance (Scor Supply Chain Operations Reference Model | Scor Framework | Apics, n.d.) de la chaine logistique selon la décomposition sur la figure suivante :

Figure 3 : Les attributs de la performance selon le modèle SCOR $®$

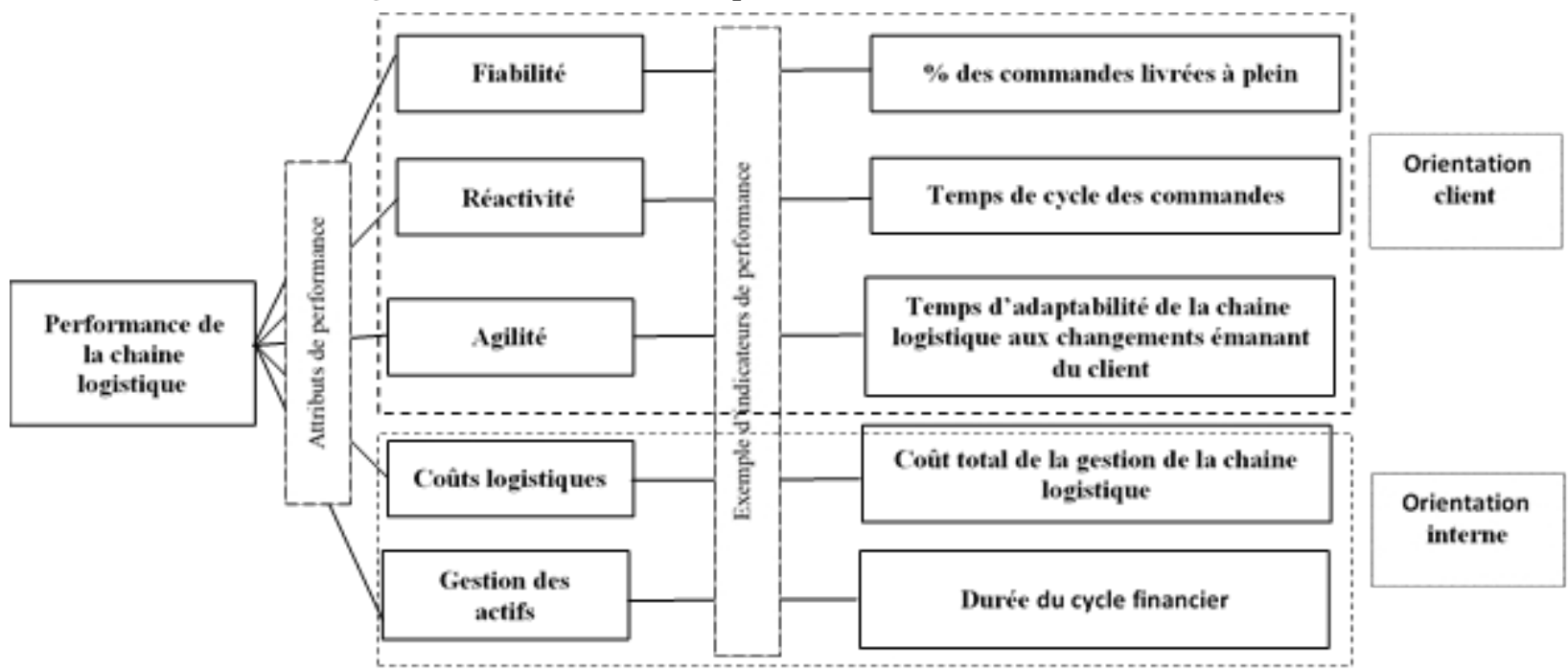

L'évaluation de la performance se fait à l'aide des attributs de la performance. Ces attributs évoquent des caractéristiques que la chaine logistique doit posséder pour être performante : fiabilité, réactivité, agilité, gestion des coûts, gestion des actifs. À chaque attribut de performance sont associés des indicateurs de performance.

La principale force de ce modèle (version $12: 2017$ ) se réside dans la capacité d'aider à la formulation d'indicateurs de performance à travers une méthodologie standardisée, adaptable à toute entreprise, pour concevoir et analyser la performance de la chaine logistique en se basant sur quatre composantes principales: processus, performance, meilleures pratiques et compétence du personnel (Lockamy et McCormack, 2004) et qui est adaptable à toute entreprise. Aussi, la crédibilité de ce modèle date de plus de 20 ans d'existence et 2000 entreprises l'ont utilisée.

Toutefois, le modèle est difficile à mettre en œuvre et nécessite une mobilisation immense des ressources humaines dédiées pour l'implémenter avec une durée d'implémentation qui avoisine les 12 mois (Scor Supply Chain Operations Reference Model | Scor Framework | Apics, n.d.), le nombre approximatif d'indicateurs proposé par le modèle est de l'ordre de 250 mesures à renseigner, sans un système d'information adéquat, cette étape est quasi-impossible (Lockamy et McCormack, 2004). 


\section{II.2. Balanced ScoreCard (BSC)}

Le modèle «Balanced ScoreCard» ou « tableau de bord équilibré » a été conçu par (Kaplan et Norton, 1996), son but est de créer un système intégré de management pour suivre la mise en œuvre de la stratégie de l'entreprise, au sujet de la chaine logistique, il permet l'évaluation de la performance de la chaine logistique à partir d'un ensemble d'indicateurs qui sont définis en fonction des objectifs stratégiques de l'entreprise.

Le «Balanced ScoreCard» organise les indicateurs de la chaine logistique en quatre perspectives illustrées sur le tableau 2.

Tableau 2: Les perspectives de BSC et ses indicateurs

\begin{tabular}{|l|ll|}
\hline Les perspectives de BSC & \multicolumn{2}{|l|}{ Exemples d'indicateurs de performance de la chaine logistique } \\
\hline 1. Perspective « Financière » & - & Retour sur investissement ; \\
& - & Pourcentage des coûts logistiques par rapport au chiffre \\
& d'affaires ; & Pourcentage des écarts par rapport au budget alloué. \\
\hline 2. Perspective «Client » & - & Nombre des nouveaux clients ; \\
& - & Réduction du temps de livraison ; \\
& - & Réduction du temps de réponse aux commandes client. \\
\hline 3. Perspective « Processus & - & Amélioration de l'efficience de la production; \\
interne» & - & Pourcentage des processus automatisés ; \\
& - & Réduction du temps de cycle/lead time. \\
\hline 4. Perspective & - & Taux de satisfaction du personnel ; \\
«Apprentissage et & - & Taux d'amélioration des compétences du personnel ; \\
croissance » & - & Utilisation de la numérisation et les activités de recherches et \\
& développement. \\
\hline
\end{tabular}

L'approche de BSC vise à créer un équilibre entre les indicateurs financiers avec d'autres indicateurs issus d'autres sources de performances : les clients, les processus et la dynamique de croissance de l'entreprise. Cette approche a une double utilité, la première est de s'assurer l'introduction, la compréhension et la traduction de la stratégie au niveau de l'ensemble des activités et des processus de la chaine logistique et la deuxième est de mobiliser les acteurs de la chaine logistique pour atteindre des objectifs des indicateurs de performance de la chaine logistique.

Toutefois, le modèle présente des limites selon (Ghalayini et Noble, 1996) et (Bititci et al, 2005) :

- La perspective «Financière » de la BSC est destinée principalement à une utilisation pour le top management composé des stratèges de l'entreprise. Elle n'est pas adaptée à une utilisation au niveau opérationnel ;

- L'absence d'une orientation réseau dans l'architecture du BSC rend difficile la traduction des objectifs stratégiques en objectifs opérationnels ;

- Le modèle ne supporte pas parfaitement la considération de la perspective de l'entreprise étendue (intégration des clients en aval et les 
fournisseurs en amont) dans l'évaluation de la performance. Cela nécessite un effort d'adaptation supplémentaire pour les utilisateurs de la BSC.

\section{II.3. ECOGRAI}

Le modèle ECOGRAI, conçue par (Bitton, 1990), vise à concevoir et à mettre en œuvre un système d'évaluation de la performance par des indicateurs en s'appuyant sur la formulation des grilles GRAI (Graphes à Résultats et Activités interreliées). Le modèle s'appuie sur une analyse descendante de l'entreprise en vue de décomposer ses objectifs stratégiques en objectifs tactiques et opérationnels avec une approche dite participative qui implique l'ensemble des utilisateurs potentiels dans la définition des indicateurs à tous les niveaux.

Le modèle ECOGRAI se base sur le triplet: objectif, mesure et variable afin de concevoir et d'implanter dans tous les centres de décision un système d'indicateurs de performance axés sur l'évaluation économique et cohérents avec l'arborescence des objectifs. Ainsi Il permet de définir un système d'indicateurs de performance cohérent et de l'intégrer dans le management, couvrant toutes ses fonctions et niveaux de décision [Ducq et al., 2003].

Le modèle ECOGRAI est développé pour assurer une cohérence entre le système d'indicateurs de performance, les variables de décisions et les objectives stratégiques et ce à travers une méthodologie d'élaboration et d'utilisation appropriée des indicateurs qui se repose sur une démarche en cinq phases présentées sur le figure 4.

Figure 4: Les étapes du modèle ECOGRAI (Adaptée de [Ducq et al., 2003])

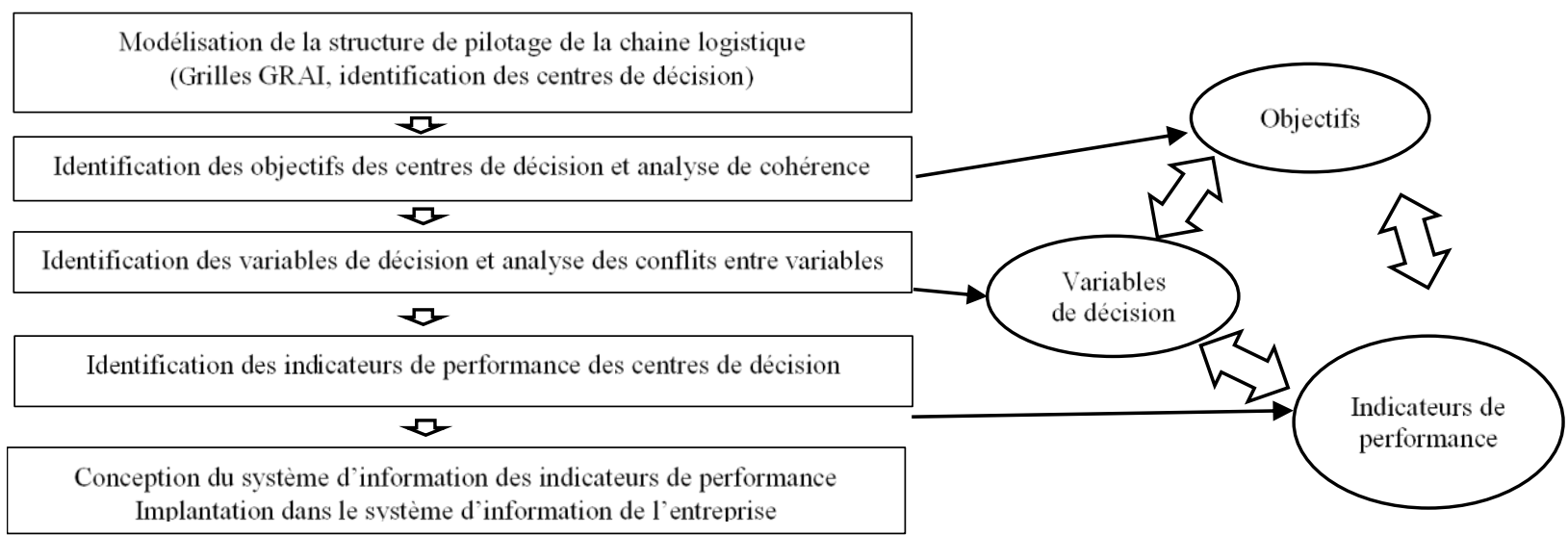

Les principales caractéristiques de la méthode ECOGRAI sont les suivantes (Charkaoui et al., 2012) : 
- La décomposition des objectifs du niveau stratégique en objectifs opérationnels ;

- L'utilisation d'outils et de supports graphiques comme les grilles GRAI et les actigrammes ;

- Une répartition cohérente des indicateurs de performance couvrant les différentes fonctions et les différents niveaux de décision (stratégique/tactique/opérationnel).

L'utilisation du modèle ECOGRAI, dans le contexte de la performance de la chaine logistique, est limitée par sa propre conception orientée à la recherche des variables de décisions (actions pour améliorer la performance) plutôt que la conception d'indicateurs de performance (variables de la performance), cette insuffisance de l'ECOGRAI dans l'évaluation de la performance est dépassée par un couplage de l'ECOGRAI avec le BSC pour assurer une évaluation complète de la performance de la chaine logistique (Lauras, 2004).

\section{II.4. ABC : Activety Based Costing}

Apparues dans les années 1980, l'ABC, le modèle des coûts par activité, est né dans les années 1987-1988, résultat des contributions parallèles du réseau Harvard et du réseau Consortium for Advanced Manufacturing International ${ }^{18}$. Pour le contexte de la chaine logistique, le modèle $A B C$ est utilisé pour l'évaluation de la performance suivant une approche par les processus (Benbba, 2009).

L'ABC établit une cartographie des processus de la chaine logistique, chaque processus délivre des prestations sur la base des activités, à des clients internes ou externes de la chaine logistique, chaque activité consomme des ressources de fonctionnement valorisées par des coûts, et chaque activité s'ajuste à un système d'indicateurs de performance (financiers et non financiers) mesurant la production de l'activité, générateurs de coûts. Ce modèle construit, à la fois, un lien de causalité entre les postes de dépenses (vision comptable) et les prestations (vision métier) et une évaluation de la performance de la chaine logistique par la mise en place d'indicateurs clés de la performance (Hofmann et Bosshard, 2017).

La contribution de l'adoption de l'ABC pour évaluer et améliorer la performance de la chaine logistique de toute entreprise est possible et significative, son adoption au niveau de petite entreprise nécessite une attention particulière en le comparant aux grandes entreprises (manufacturières ou non manufacturières) et son adoption au niveau des

\footnotetext{
${ }^{18}$ Consortium for Advanced Manufacturing International est une organisation de recherche et de développement à laquelle participent de grands groupes industriels, des agences gouvernementales américaines, des institutions publiques, des cabinets d'audit, des universitaires et des associations.
} 
entreprises non manufacturières révèle un degré supérieur de difficulté par rapport à des entreprises manufacturières (Hofmann et Bosshard, 2017).

L'utilisation du modèle $\mathrm{ABC}$, dans l'évaluation de la performance de la chaine logistique, est confrontée à des coûts élevés de la mise en œuvre de l'ABC liés essentiellement à la réorganisation de la gestion pour prendre en compte l'aspect processus, logiciels spéciaux, équipements de collecte et de traitement de données, formation du personnel et aussi à ce que l'aspect comptable, qui est l'origine du modèle $\mathrm{ABC}$, ne peut pas caractériser à lui seul une évaluation de la performance de la chaine logistique.

\section{Discussions et comparaison}

La section précédente a présenté un aperçu sur les modèles de référence d'évaluation de la performance de la chaine logistique (SCOR, BSC, ECOGRAI et ABC) en se basant sur une étude et une analyse bibliographique qui a permis de déduire qu'aucun modèle n'est complet. Chacun peut être amélioré en s'inspirant d'autres modèles existants. Aussi un couplage entre deux modèles est possible, sur le plan théorique, pour dépasser les limites de chaque modèle et en conséquence pour assurer une évaluation optimale de la performance de la chaine logistique.

Ainsi, les quatre modèles exploités se reposent sur un système d'indicateurs de performance de la chaine logistique sans proposer une méthodologie d'élaboration d'un plan d'action pour atteindre les objectifs de ce système.

Par ailleurs, il est à préciser que la performance globale de chaque modèle dans l'évaluation de la performance de la chaine logistique dépend intrinsèquement de son implémentation, son dégrée d'utilisation optimale par l'entreprise-utilisatrice.

Le tableau suivant résume une comparaison entre les quatre modèles de référence de cet article sur la base de huit critères retenus par (Lauras, 2004).

Tableau 3: Comparaison des modèles de références

\begin{tabular}{|l|l|l|l|l|}
\hline \multicolumn{2}{|c|}{ Les critères de comparaison } & \multicolumn{4}{l|}{ Les modèles de référence } \\
\cline { 2 - 5 } & SCOR $^{\circledR}$ & BSC & ECOGRAI & ABC \\
\hline $\begin{array}{l}\text { Définition des indicateurs de performance en se basant sur } \\
\text { les déterminants de la performance de la chaine logistique }\end{array}$ & Oui & Oui & Non & Oui \\
\hline $\begin{array}{l}\text { Association des indicateurs de performance aux activités de } \\
\text { l'ensemble des processus de la chaine logistique }\end{array}$ & Oui & Oui & Oui & Non \\
\hline $\begin{array}{l}\text { Association des indicateurs de performance aux centres de } \\
\text { décision sur la chaine logistique }\end{array}$ & Oui & Oui & Oui & Oui \\
\hline Cohésion temporelle des indicateurs & Non & Non & Oui & Non \\
\hline Cohérence fonctionnelle des indicateurs & Oui & Oui & Oui & Oui \\
\hline l'approche agrégative des indicateurs & Non & Non & Non & Oui \\
\hline Identification directe des causes de la (non-)performance & Non & Non & Non & Non \\
\hline Elaboration d'un plan actions & Non & Non & Non & Non \\
\hline
\end{tabular}




\section{Conclusion}

Une panoplie de modèles d'évaluation de la performance de la chaine logistique a été développée pour suivre l'évolution conceptuelle de la chaine logistique. En l'absence de consensus autour d'un modèle commun de l'évaluation de la performance de la chaine logistique, cet article se limite à 1'exploitation théorique des modèles $\left(\mathrm{SCOR}^{\circledR}, \mathrm{BSC}, \mathrm{ECOGRAI}\right.$ et $\left.\mathrm{ABC}\right)$ dits de références à ce sujet. Pour chaque modèle, des limites théoriques ont été révélées sans mener une comparaison objective sur une base commune entre ces modèles de références. Il est à préciser que ces modèles ont été désignés pratiquement pour évaluer la performance de l'entreprise et ne sont pas conçus spécialement pour évaluer la performance de la chaine logistique.

On peut avancer que les quatre modèles exploités ne permettent pas de supporter l'élaboration d'un plan d'action et d'identifier les sources probables de la performance ou de la non-performance. D'ailleurs, on a constaté que chacun des modèles n'assure que quatre critères de comparaison, sur les huit critères de base ce qui a rendu la comparaison difficile. On a pu relever que chacun des modèles œuvre à l'évaluation de la performance de la chaine logistique différemment, mais si les deux critères de comparaison majeurs (la détermination des indicateurs et l'association des indicateurs avec l'ensemble des processus de la chaine logistique) sont seuls retenus et les plus à même d'évaluer la pertinence de la chaine logistique, les modèles: Balanced ScoreCard et $\mathrm{SCOR}^{\circledR}$ sont les plus éligibles à se charger de l'évaluation de la chaine logistique.

Ce travail devra être complété par une exploitation pratique de ces modèles par une étude empirique dans les entreprises. Aussi, il est jugé pertinent d'envisager un travail de recherche pour évaluer l'opportunité de combiner deux à trois modèles dans un nouveau modèle d'évaluation de la performance pour surmonter les limites de chaque modèle précité.

\section{References:}

1. Abu-Suleiman, A., Boardman, B., \& Priest, J. W. (2004). A Framework for an Integrated Supply Chain Performance Management System. Industrial Engineering Research Conference.

2. Avelar-Sosa, L., García-Alcaraz, J. L., \& Maldonado-Macías, A., A. (2019). Evaluation of Supply Chain Performance : A Manufacturing Industry Approach. Springer.

3. Beamon, B., M. (1998). Supply chain design and analysis: Models and methods. International Journal of Production Economics, 55(ue 3), 281-294.

4. Benbba, B. (2016). Evaluation de la performance des chaines logistiques: Une approche par les processus. Revue Marocaine de Gestion et d'Economie, 1. 
5. Bititci, U. S., Mendibil, K., Martinez, V., \& Albores, P. (2005). Measuring and managing performance in extended enterprises. International Journal of Operations \& Production Management, 25(4), 333-353. https://doi.org/10.1108/01443570510585534

6. Bitton, M. (1990). ECOGRAI: Méthode de conception et d'implantation de systèmes de mesure de performance pour organisations industrielles.

7. Brulhart, F., \& Moncef, B. (2010). L'impact des pratiques de SCM sur la performance de l'entreprise : Une étude empirique dans le contexte français. Finance Contrôle Stratégie, 13(1), 33.

8. Charkaoui, A., Ait Ouahman, A., \& Bouayad, B. (2012). Application of ECOGRAI/BSC method for controlling logistic performance : Case of a Moroccan clothing company. International Journal of Business, Humanities and Technology, 2(2), 26-35.

9. Derujinsky-Laguecir, A., Kern, A., \& Lorino, P. (2011). Une approche instrumentale des indicateurs de performance. Management \& Avenir, 42(2), 111. https://doi.org/10.3917/mav.042.0111

10. Qing-ling, D., Shu-min, C., Lian-liang, B., \& Jun-mo, C. (2008). Application of pdca cycle in the performance management system. 2008 4th International Conference on Wireless Communications, Networking and Mobile Computing. https://doi.org/10.1109/WiCom.2008.1682

11. Ducq, Y., Gentil, M.H., Merle, C., \& Doumeingts, G. (2003). Conception et implantation de systèmes d'indicateurs de performance. Dans Tahon, C., Evaluation des performances des systèmes de production, Hermès.

12. Estampe, D., Lamouri, S., Paris, J.-L., \& Brahim-Djelloul, S. (2013). A framework for analysing supply chain performance evaluation models. International Journal of Production Economics, 142(2), 247258. https://doi.org/10.1016/j.ijpe.2010.11.024

13. Finet, A. (2009). Gouvernance d'entreprise: Nouveaux défis financiers et non financiers. De Boeck.

14. Fitzgerald, L., Johnson, R., Brignall, S., R., S., \& Voss, C. (1991). Performance Measurement in Service Business. CIMA.

15. Ghalayini, A. M., \& Noble, J. S. (1996). The changing basis of performance measurement. International Journal of Operations \& Operations Management, 16(8), 63-80.

16. Gunasekaran, A., \& Kobu, B. (2007). Performance measures and metrics in logistics and supply chain management: A review of recent literature (1995-2004) for research and applications. International Journal of Production Research, 45(12), 2819-2840. https://doi.org/10.1080/00207540600806513 
17. Gunasekaran, A., Patel, C., \& McGaughey, R. E. (2004). A framework for supply chain performance measurement. International Journal of Production Economics, $87(3)$

$333-347$. https://doi.org/10.1016/j.ijpe.2003.08.003

18. Ho, D. C. K., Au, K. F., \& Newton, E. (2002). Empirical research on supply chain management: A critical review and recommendations. International Journal of Production Research, 40(17), 4415-4430. https://doi.org/10.1080/00207540210157204

19. Hofmann, E., \& Bosshard, J. (2017). Supply chain management and activity-based costing: Current status and directions for the future. International Journal of Physical Distribution \& Logistics Management, 47(8), 712-735. https://doi.org/10.1108/IJPDLM-042017-0158

20. Holmberg, S. (2000). A systems perspective on supply chain measurements. International Journal of Physical Distribution \& Logistics Management, 30(10), 847-868. https://doi.org/10.1108/09600030010351246

21. Huang, S. H., Sheoran, S. K., \& Keskar, H. (2005). Computer-assisted supply chain configuration based on supply chain operations reference (Scor) model. Computers \& Industrial Engineering, 48(2), 377-394. https://doi.org/10.1016/j.cie.2005.01.001

22. Kaplan, R. S., \& Norton, D. P. (1996). Strategic learning \& the balanced scorecard. Strategy \& Leadership, 24(5), 18-24. https://doi.org/10.1108/eb054566

23. Kusrini, E., Subagyo, \& Masruroh, N. A. (2014). Good criteria for supply chain performance measurement. International Journal of Engineering Business Management, 6, 9. https://doi.org/10.5772/58435

24. Lai, K., Ngai, E. W. T., \& Cheng, T. C. E. (2002). Measures for evaluating supply chain performance in transport logistics. Transportation Research Part E: Logistics and Transportation Review, 38(6), 439-456. https://doi.org/10.1016/S13665545(02)00019-4

25. Lappide, L. (2002). What about measuring Supply Chain Performance. ASCET, 3.

26. Lauras, M. (2004). Méthodes de diagnostic et d'évaluation de performance pour la gestion de chaines logistiques : Application à la coopération maison-mère, filiales internationales dans un groupe pharmaceutique et cosmétique.

27. Cheyroux, L. (2003). Sur l'évaluation de performances des chaines logistiques. 
28. Lebas, M. (1995). Oui, il faut définir la performance. Revue Française de Comptabilité, 66-71.

29. Lee, H. L., \& Billington, C. (1992). Managing supply chain inventory : Pitfalls and opportunities. Sloan Management Review, 33(3), 65-73.

30. Lockamy, A., \& McCormack, K. (2004). Linking SCOR planning practices to supply chain performance: An exploratory study. International Journal of Operations \& Production Management, 24(12), 1192-1218. https://doi.org/10.1108/01443570410569010

31. Lorino, P. (2003). Méthodes et pratiques de la performance: Le pilotage par les processus et les compétences. Éditions d'Organisation.

32. Marr, B. (2006). Strategic performance management: Leveraging and measuring your intangible value drivers (1. ed., reprinted). Elsevier, Butterworth-Heinemann.

33. Morgan, C. (2007). Supply network performance measurement: Future challenges? The International Journal of Logistics Management, 18(2), 255-273. https://doi.org/10.1108/09574090710816968

34. Neely, A., Gregory, M., \& Platts, K. (1995). Performance measurement system design: A literature review and research agenda. International Journal of Operations \& Production Management, 15(4), 80-116. https://doi.org/10.1108/01443579510083622

35. Rafele, C. (2004). Logistic service measurement: A reference framework. Journal of Manufacturing Technology Management, 15(3), 280-290. https://doi.org/10.1108/17410380410523506

36. Ramaa, A., Rangaswamy, T. M., \& Subramanya, K. N. (2009). A review of literature on performance measurement of supply chain network. 2009 Second International Conference on Emerging Trends in Engineering Technology, 802-807.

https://doi.org/10.1109/ICETET.2009.18

37. Salgado, M. (2013). La performance : Une dimension fondamentale pour l'évaluation des entreprises et des organisations. https://hal.archives-ouvertes.fr/hal-00842219

38. Scor supply chain operations reference model | scor framework | apics. (n.d.). Retrieved May 7, 2020, from http://www.apics.org/apicsfor-business/frameworks/scor

39. Waters, C. D. J., \& Chartered Institute of Logistics and Transport in the UK (Eds.). (2007). Global logistics: New directions in supply chain management (5th ed). Kogan Page Ltd. 